\title{
Comparative study of three herbal medicine formulas on high fat diet-induced obese mice
}

\author{
Hyo-Nam Song ${ }^{1}$ and Kim Jeungbeum ${ }^{2}$ \\ ${ }^{1}$ Department of Oriental Medical Food and Nutrition, Semyung University and ${ }^{2}$ College of Oriental Medicine, \\ Semyung University, Jechon 390-711, South Korea
}

Objectives: The purpose of this study was to compare the anti-obese effects of three kinds of newly prescribed herbal medicine formulas on the high-fat diet induced obese mice.

Methodology: Six kinds of water extracts at high (DS-H, TE-H, YK-H) and low (DS-L, TE-L, YK-L) concentration from the three prescription of Dansam-gamibang (DS), Taeum-gamibang (TE) and Yukgye-gamibang (YK), which were composed of five, seven and five kinds of Korean herbal medicine, respectively, were administered once a day in C57BL mice for 6 weeks.

Experimental mice were randomly divided into eight groups: normal control group (Con, $n$ 15), high fat diet control group (HFD, $n$ 15), six treatment groups with high concentration of $500 \mathrm{mg} / \mathrm{kg}$ (DS-H, TE-H, YK-H, $n$ 15) and low concentration of $250 \mathrm{mg} / \mathrm{kg}$ (DS-L, TE-L, YK-L, $n$ 15).

Data and Results: Six-weeks of feeding with HFD resulted in significant increase in body weight compared with the normal control group. The level of total cholesterol (TC) and LDL-cholesterol were not significantly decreased in experimental groups than normal control group $(P<0.05)$. Significant differences at HDL-cholesterol level was not shown by the six herbal medicine formulas, either. In contrast, triglyceride (TC) level was markedly decreased in DS-L [24.92 (SD 1.59)], DS-H [25.00 (SD 1.65)], YK-L [24.62 (SD 1.82)] and YK-H [21.46 (SD 1.73)] compared with HFD group [32.64 (SD 2.36)] and normal control group [52.93 (SD 4.05)](P<0.05). YK extract was found to lower the leptin level effectively both low concentration group [27.53 (SD 2.15)] and high concentration group (20.39 (SD 1.80 ) compared with HFD group [32.06 (SD 3.28)] $(P<0.05)$.

Conclusion: These results suggested that the Yukgye-gamibang extracts have the best anti-obese effect in high-fat diet induced obesity mice among three herbal medicine formulas. Thus this new formula is expected to be a novel agent for many functional foods in Korea.

1. Whang JP \& Yun IJ (2010) The anti-obesity effects of gamibang 4 on obesity-induced mice. J Korean Oriental Med 31, 30-46.

2. Seo DJ, Kim JM, Kim TH et al. (2010) Anti-obesity effects of foeniculum fructus water extract. J Korea Food Nutr Sci 39, 1604-1610.

3. Hong-Tae Kim, Dae-Dong Kim, Sae-Kwang Ku et al. (2010) Anti-obesity effects of artemisiae capillaris herba, artemisia capillaris stem aqueous extracts on the high fat diet supplied mice. J Vet Clin 27, 348-365. 